\title{
Osteomielite de Garré: Relato de caso clínico
}

\section{Garre's osteomyelitis: Case report}

\author{
Jordana Silva Mendes Barros \\ Cirurgiã Dentista graduada pela Pontifícia Universidade Católica de Minas Gerais. Belo Horizonte, MG, Brasil. \\ SÂMILA GonçALVES BARRA* \\ Especialista em Radiologia e Imaginologia Odontológica pela Universidade \\ Federal do Rio de Janeiro. Rio de Janeiro, RJ, Brasil. \\ Ana Márcia Viana Wanzeler \\ Especialista em Radiologia e Imaginologia Odontológica pela Universidade \\ Federal do Rio de Janeiro. Rio de Janeiro, RJ, Brasil. \\ FLÁVIO RICARDO MANZI \\ Professor Adjunto da Radiologia e Professor. Coordenador do mestrado em Radiologia da e da Residência em \\ Radiodiagnóstico da Pontifícia Universidade Católica de Minas Gerais. Belo Horizonte, MG, Brasil.
}

\begin{abstract}
RESUMO
Introdução: a osteomielite de Garré, também conhecida como periostite ossificante, é um tipo raro de lesão que afeta, principalmente, a primeira e a segunda décadas de vida, sem predileção por sexo e raça, e acomete, em sua grande maioria, a mandíbula, em região de molares. O fator etiológico mais comum é a infecção ou irritação leve a moderada, sendo a cárie na região de molar permanente a mais relacionada. Radiograficamente, a periostite ossificante é caracterizada pela presença de esclerose densa com nova formação óssea do periósteo, havendo dupla formação óssea, resultando em uma aparência de "casca de cebola" em razão da sucessiva deposição de camadas de osso subperiósteo. Objetivo: relatar um caso de osteomielite de Garré com acompanhamento pós-remoção do agente agressor. Caso clínico: paciente do sexo feminino, 9 anos de idade, apresentando febre e fístula extraoral na região da mandíbula, no lado esquerdo da face, sem sintomatologia dolorosa e com assimetria facial. Foram realizadas radiografias panorâmica e periapical, constatando cárie envolvendo toda a coroa dentária, com rarefação óssea periapical difusa compatível com lesão periapical. Apresentava, ainda, osteogênese reacional adjacente à rarefação óssea, convergindo para o diagnóstico de osteomielite de Garré. Conclusão: o diagnóstico de osteomielite de Garré é essencialmente clínico e radiográfico. O prognóstico é favorável quando a causa é removida totalmente e realizada antibioticoterapia coadjuvante.
\end{abstract}

Palavras-chave: osso; infecção; osteomielite; diagnóstico; patologia.

\begin{abstract}
Introduction: Garré's osteomyelitis, also known as periostitis ossificans, is a rare type of injury that affects mainly the first and second decades of life, without predilection for sex and race, and affects mostly the jaw in the molar area. The most common etiologic factor is mild to moderate infection or irritation, related mostly with cavities in the permanent molar region. Radiographically, ossifying periostitis is characterized by the presence of dense sclerosis with new periosteal bone formation, with double bone formation, resulting in an "onion skin" appearance due to the successive deposition of layers of subperiosteal bone. Objective: To report a case of Garré's osteomyelitis with post-removal monitoring of the offending agent. Clinical case: A 9-year old female patient, with fever and extra-oral fistula in the left side of the jaw with painless symptoms and facial asymmetry. Panoramic and periapical radiographs showed cavities involving the whole dental crown with diffuse periapical bone rarefaction compatible apical periodontitis. She also presented, reactional osteogenesis adjacent to the bone converging to the diagnosis of Garré's osteomyelitis. Conclusion: The diagnosis of Garré's osteomyelitis is essentially clinical and radiographic. The prognosis is favorable when the cause is completely removed and adjunct antibiotic therapy is held.
\end{abstract}

Keywords: bone; infection; osteomyelitis; diagnosis; pathology. 


\section{INTRODUÇÃO}

Osteomielite de Garré é uma doença inflamatória rara, de caráter crônico, marcada por reação periosteal, que induz uma neoformação óssea. Acomete principalmente a região da mandíbula e, em casos mais raros, pode localizar-se na região metafisária dos ossos longos. ${ }^{1} \mathrm{~A}$ doença também é conhecida como osteomielite esclerosante de Garré (OEG), osteomielite crônica com periosteíte proliferativa, osteomielite esclerosante crônica, periostite ossificante e osteomielite esclerosante crônica não supurativa. A variedade de nomes para essa doença reflete o grande número de especulações sobre sua etiologia e/ou fisiopatologia. ${ }^{2}$

A osteomielite de Garré, na maioria dos casos, é de origem osteogênica, tendo como fator mais comum a cárie em região de molar permanente. $^{3}$ Outros fatores podem ser incluídos, como infecção periapical dos molares inferiores, infecção periodontal, fraturas não tratadas, desenvolvimento do folículo do dente, dentes inclusos, exodontia recente, cisto bifurcado vestibular, cisto odontogênico lateral, infecção não odontogênica e origem idiopática. ${ }^{4}$

Os aspectos clínicos da osteomielite de Garré são representados pela assimetria facial, sustentados por uma grande tumefação de consistência rígida, localizada e unilateral, que pode ser vista em exames intra e extra orais. ${ }^{5}$ Episódios de dor leve a moderada também podem acontecer ocasionalmente, com períodos de latência desta e aumento progressivo da sintomatologia. A condição de trismo também pode ocorrer, assim como a linfodenopatia, febre e mal-estar. ${ }^{2,6}$

O exame clínico é muito importante durante o processo de diagnóstico. O exame radiológico inclui radiografia panorâmica, periapical, radiografias oclusais, tomografia computadorizada e cintilografia. ${ }^{7}$ Os aspectos radiográficos também se apresentam com características bemespecíficasparaaresolução do caso, possibilitando melhor diagnóstico e, consequentemente, o prognóstico da doença. ${ }^{8}$ A resposta periostal pode ser observada na radiografia oclusal sob a forma de laminações concêntricas, características desta doença, que geralmente aparecem paralelas umas às outras e ao longo do eixo do osso afetado, dando o aspecto conhecido como "casca de cebola".,8 Os casos mais comuns dessa patologia podem ser concluídos pelo clínico radiográfico, porém alguns diagnósticos devem ser confirmados com exames histológicos. ${ }^{9}$ Existem enfermidades que apresentam características radiográficas semelhantes às da osteomielite de Garré e precisam ser diferenciadas; dentre elas: a síndrome de Caffey (hiperostose cortical infantil), que, além dos ossos faciais, pode acometer outros ossos, como clavículas, pélvis e as extremidades; o sarcoma de Ewing, que exibe um crescimento rápido acompanhado de nevralgia e parestesia, associando-se a lesões osteolíticas e difusas, com predileção por homens na terceira década de vida, com sinais de febre e tumefação local, seguidas de imagens que lembram "casca de cebola" ou "raios de sol" ou "roído de traça"; a hipervitaminose $A$, que não causa tumefação facial, e a displasia fibrosa, que apresenta aspecto radiográfico de "vidro fosco". 2,10,11

O tratamento constitui-se na remoção do fator etiológico, assim como cobertura antibiótica profilática. A resposta normalmente é satisfatória, com redução da tumefação, embora possa permanecer alguma expansão óssea residual após algum tempo. $O$ prognóstico da osteomielite de Garré é favorável, sendo a remoção do fator causal da infecção a resolução do caso. ${ }^{10}$

\section{Caso clínico}

Paciente do sexo feminino, 9 anos de idade, procurou a Faculdade de Odontologia da Pontifícia Universidade Católica de Minas Gerais (PUC-Minas) apresentando febre e 
fístula extraoral na região de mandíbula, no lado esquerdo da face, sem sintomatologia dolorosa e com assimetria facial (Figura 1).

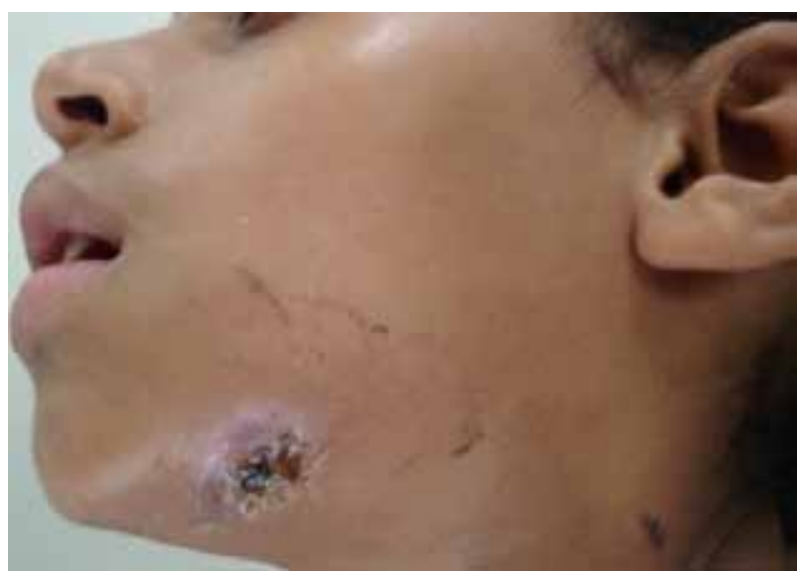

Figura 1 - Fístula extra oral

Relatou que três meses antes haviam ocorrido sucessivos edemas na região acompanhados de dor e febre. No exame clínico foi observada lesão cariosa extensa, destruindo quase a totalidade da coroa do dente 36 . Foram realizadas radiografias panorâmica e periapical deste dente, no qual foi constatada cárie envolvendo toda a coroa dentária com rarefação óssea periapical difusa compatível com lesão periapical (abscesso periapical crônico). Verificou-se, ainda, osteogênese reacional adjacente à rarefação óssea (Figura 2). Em virtude do quadro clínico (presença

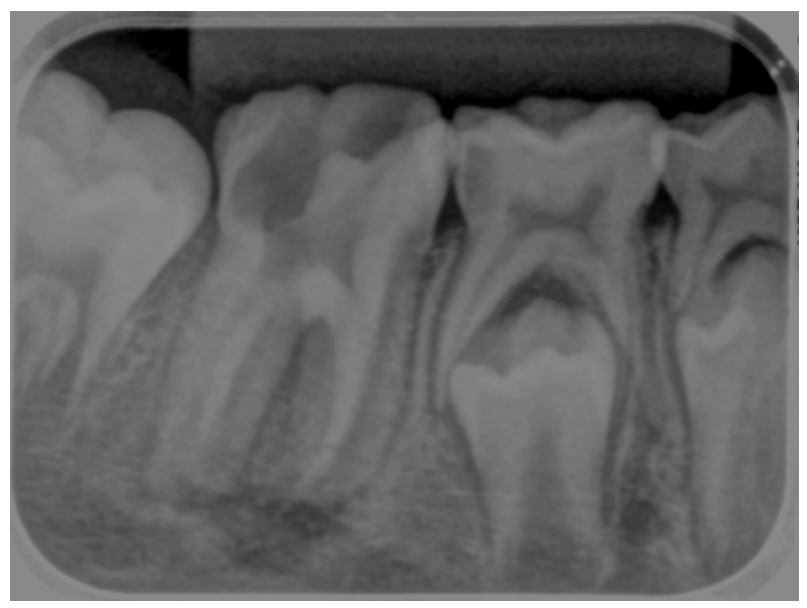

Figura 2 - Radiografia periapical evidenciando osteogênese reacional adjacente à rarefação óssea de fístula extraoral), também foi realizada a radiografia oclusal (Figura 3 ), na qual se observou duplicação óssea com aspecto de casca de cebola. Assim, tanto as evidências clínicas como as radiográficas convergiram para o diagnóstico de osteomielite de Garré.

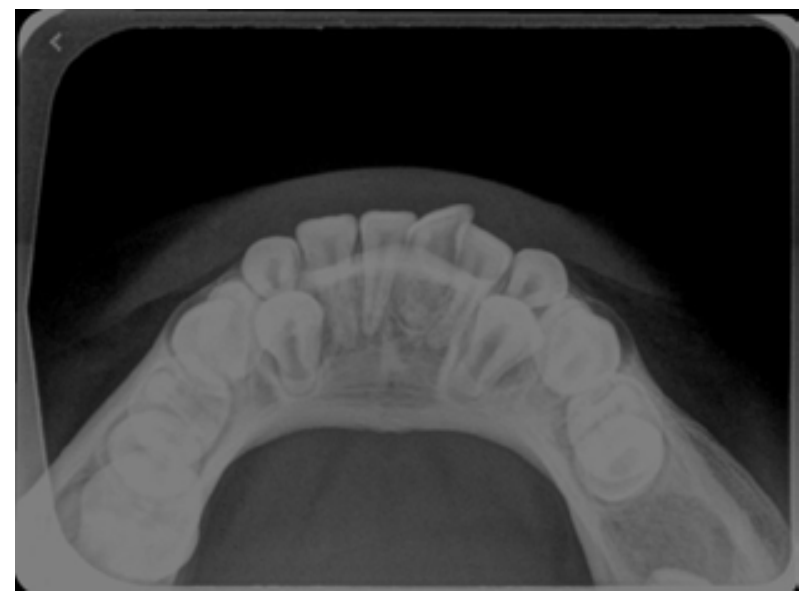

Figura 3 - Radiografia oclusal evidenciando duplicação óssea com aspecto de casca de cebola

O tratamento incluiu a exodontia do dente afetado pela periostite ossificante. Após o procedimento, houve regressão do quadro de infecção, com diminuição da rarefação óssea e lesão periapical, que pode ser visualizada pela radiografia periapical e pela radiografia panorâmica (Figuras 4 e 5).

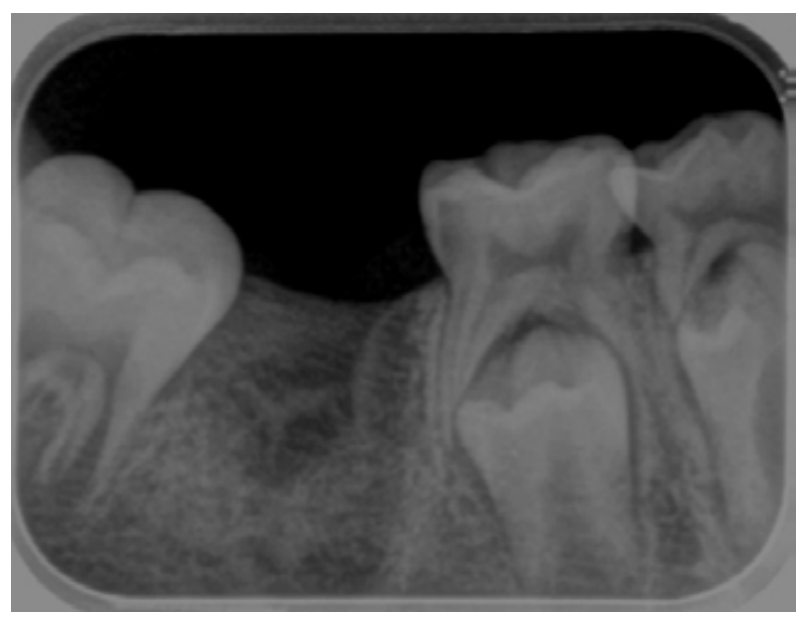

Figura 4 - Radiografia periapical mostrando a regressão do quadro de infecção. 


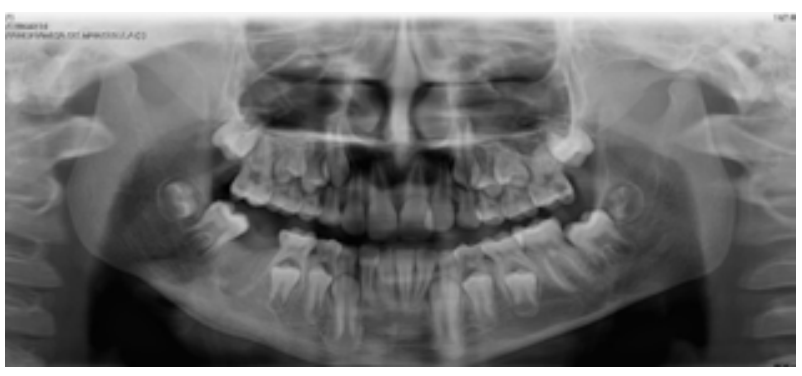

Figura 5 - Radiografia panorâmica mostrando a regressão do quadro de infecção

\section{Discussão}

Osteomielite de Garré é uma resposta periosteal exuberante a uma inflamação, caracterizada por uma fase aguda, podendo afetar pessoas com menos de 25 anos de idade, tendo maior predileção pela mandíbula, surgimento rápido, dor, tumefação dos tecidos moles adjacentes, febre e linfadenopatia., 2,11,12 O caso clínico relatado ocorreu em uma criança, em concordância com a literatura consultada, que traz a maioria dos casos com a mesma década de vida. Não se sabe ao certo o que provoca esse tipo de reação no tecido ósseo, porém algumas hipóteses são consideradas, como a alta atividade do periósteo na criança, que reagiria ante uma infecção de baixa virulência, ou como resultado do desequilíbrio entre as atividades osteoblásticas e osteoclásticas, com predominância da primeira, determinada por fatores individuais..$^{8,13,14}$ Sabe-se que o tecido ósseo de indivíduos jovens reage com muito mais dinâmica que em indivíduos de mais idade. Quanto à localização, o caso relatado no presente artigo ocorreu na mandíbula, também em concordância com a literatura, que indica maior ocorrência nessa região. Na maioria dos casos, a patologia apresenta-se unifocal e unilateral, ${ }^{9}$ como pode-se observar no caso clínico relatado. Entretanto, já foram relatados casos envolvendo os quatro quadrantes..$^{2,8}$ A maxila é raramente sítio de processo osteomielítico, identificando-se apenas um caso descrito em maxila adulta. ${ }^{7}$
Além da predileção por mandíbulas de crianças, a osteomielite de Garré é usualmente descrita na literatura como uma infecção associada ao primeiro molar inferior permanente acometido por cárie. ${ }^{3,11}$ A preferência por esse dente talvez possa ser explicada pelo fato de que o primeiro molar inferior é um dos primeiros dentes permanentes a irromper na cavidade bucal, consequentemente, o que ficaria mais tempo exposto a agentes cariogênicos, favorecendo a proliferação bacteriana. ${ }^{5,9}$ Além disso, há relatos sobre a possibilidade da ocorrência estar associada à doença periodontal e outros fatores, como exodontias recentes, traumas e alterações metabólicas sob a forma reacional do organismo ante uma agressão de longa frequência de atuação, de caráter crônico e não supurativo. ${ }^{7,14}$

O presente relato de caso reforça que - clínico e radiográfico estabelecem o diagnóstico da osteomielite de Garré, em sua apresentação mais comum. Apesar de não ser isoladamente conclusivo, o exame radiográfico é de grande importância no processo de diagnóstico, sendo que as radiografias oclusal e panorâmica supriram a necessidade do diagnóstico sem a necessidade de exame radiológico complementar em três dimensões ou tomografia computadorizada de feixe cônico. No caso clínico apresentado, o tratamento proposto foi a remoção da causa, seguido de antibioticoterapia, sendo que esse tipo de terapêutica é recomendado pela literatura.

Estudos mostram que $82,7 \%$ das periosteítes ocorrem abaixo da borda da mandíbula, $43 \%$ no córtex bucal e $6,5 \%$ no córtex lingual. Este tipo de reação óssea está associado a um processo inflamatório infeccioso crônico. ${ }^{11,15}$ No caso relatado, a demora na procura pelo tratamento foi a causa principal da evolução da doença, incluindo desenvolvimento de abscesso e posterior formação de fístula extraoral, caracterizando uma condição crônica. 


\section{Conclusão}

Podemos concluir que o diagnóstico de osteomielite de Garré é, essencialmente, clínico e radiográfico, considerando-se a análise radiográfica imprescindível para a determinação do diagnóstico. Só há necessidade da confirmação histológica em casos nos quais surjam dúvidas, quando a imagem radiográfica não mostra claramente a expansão da cortical em camadas e não apresenta necrose pulpar e lesão periapical associada.

O prognóstico é favorável quando a causa é removida totalmente e realizada antibioticoterapia coadjuvante.

\section{REFERÊNCIAS}

1 Oulis, C, Berdousis E, Vadiakas G, Goumenos G. Garre's osteomyelitis of an unusual origin in a 8 year old child: A case report. Int J Pediatr Dent. 2000; 10(3):240-4.

2 Moares, FB, Motta TMV, A Severin AA, et al. Garré's sclerosing osteomyelitis: case report. Rev Bras Ortop. 2014; 49(4): 401-404.

3 Belli E, Matteini C, Andreano T. Sclerosing osteomyelitis of Garré periostitis ossificans. J Craniofac Surg. 2002; 13(6):765-8.

4 Kadom N, EgloffA, Obeid G, BandarkarA, Vezina G. Juvenile mandibular chronic osteomyelitis: multimodality imaging findings. Oral Surg Oral Med Oral Pathol Oral Radiol Endod. 2011; 111(3):38-43.

5 Kannan SK, Sandhya G, Selvarani R. Periostitis issificans (Garré's osteomyelitis) radiographic study of two cases. Int J Paediatr Dent. 2006; 16: 59-64.

6 Akiyama K, Kyoko O, kondo H, Kobayashi J, Yoshimura H, Tobita T. Root-end resection for preservation of the causative molar in Garrè's osteomyelitis of the mandible: A case report. J Oral Maxillofac Surg Med Pathol. 2013; 25(2): 139-42.

7 Obel, G, Krogdahl A, Thygesen T, Godballe C. Juvenile mandibular chronic osteomyelitis: 3 cases and a literature review. J Oral Maxillofac Surg. 2013; 71(2): 305-9.
8 Silva, MM, Castro, AL; Castro, EVFL; Coclete , Gilberto A. Garré' s osteomyelitis. An update and report of two new cases. Rev Bras Odontol. 2009; 66(1): 8-11.

9 Belli E, Matteini C, Andreano T. Sclerosing osteomyelitis of Garré periostitis ossificans. J Craniofac Surg. 2002; 13(6): 765-68.

10 Bossche LHV, Demeulemeester JDA, Bossuyt $\mathrm{MH}$. Periodontal infection leading to periostitis ossificans (Garrés osteomyelitis) of the mandible. Report of case. J Periodontol. 1993; 64(1): 60-2.

11 Conte N, Gonçalves M, Pereira Filho VA; Carvalho WRS de, Castro-Silva LM de. Osteomielitis Crónica con Periostitis Proliferativa. International Journal of Odontostomatology. 2013; 7(1): 5-10.

12 Nortje CJ, Wood RE, Grotepass F. Periostitis ossificans versus Garrè's osteomyelitis. Part II: Radiologic analysis of 93 cases in the jaws. Oral Surg Oral Med Oral Pathol. 1988; 66(2): 249-60.

13 Eisenbud L, Miller J, Roberts IL. Garrè's proliferative periostitis occurring simultaneously in four quadrants of the jaws. Oral Surg Oral Med Oral Pathol. 1981; 51: 172-8.

14 Vieira CL, Ayres de Melo VER. Osteomyelitis story of clinical case. Int. J. Dent. 2006; 1(1): 35-40.

15 Castro FP, Guaré R de O, Mathias RS. Garré's osteomyelitis: General considerations and report of a case. Rev ABO Nac. 2001; 9(4): 247-50.

Endereço para correspondência:

Sâmila Gonçalves Barra

Av. Dom José Gaspar, Prédio 45 - Clínica de

Radiologia

Coração Eucarístico, Belo Horizonte - MG, Brasil

CEP: 30535-901

Fones: 31 - 8421-6607 // $31-33816389$

samilagbarra@gmail.com

Submetido em: 29-3-2015

Aceito em: 1-6-2015 